\title{
IN-SITU NANOINDENTATION - A UNIQUE PROBE OF DEFORMATION RESPONSE IN MATERIALS
}

\author{
A.M. Minor ${ }^{1}$, E.T. Lilleodden ${ }^{1}$, M. Jin ${ }^{1,3}$, E.A. Stach ${ }^{2}$, D. Chrzan ${ }^{1,3}$, J.W. Morris, Jr. ${ }^{1,3}$ T.A. \\ Friedmann $^{4}$, X. Xiao ${ }^{5}$, O.H. Auciello ${ }^{5}$, and J.A. Carlisle ${ }^{5}$ \\ 1 Materials Science Division, Lawrence Berkeley National Laboratory, Berkeley, CA 94720 \\ 2 National Center for Electron Microscopy, Lawrence Berkeley National Laboratory, Berkeley, CA 94720 \\ 3 Department of Materials Science and Engineering, University of California, Berkeley, CA 94720 \\ 4 Department of Nanostructure and Semiconductor Physics, Sandia National Laboratory, Albuquerque, NM \\ 5 Materials Science Division, Argonne National Laboratory, Argonne, IL
}

In-situ transmission electron microscopy is particularly well suited to observing the mechanisms of dislocation nucleation, propagation and interaction in materials. We have built a novel specimen holder that mimics the now standard ex-situ characterization technique of nanoindentation, and allows us to controllably introduce deformation in a material while simultaneously observing the process in real time in the microscope..$^{1-3}$

In this presentation, we will present a number of recent observations of the deformation behavior of aluminum thin films on silicon, single crystal silicon and silicon substrates coated with 'diamondlike carbon' thin films.

In the case of aluminum thin films on silicon, we clearly demonstrate that at the point of elastic instability dislocations are nucleated underneath the indenter tip, and glide through the film to the film substrate interface and grain boundaries. ${ }^{3}$ Similarly, it will be shown that during indentation of metal films, there is a critical grain size below which the films undergo a 'softening' due to easy nucleation of dislocations at the grain boundaries. ${ }^{4,5}$

Indentation of single crystal silicon in our particular experiments results in particularly surprising behavior. Unlike observations of bulk indentation - where phase transformation of different types are observed ${ }^{6}-$ we find that deformation proceeds by the nucleation and glide of dislocations from the top surface into the sample bulk, without any phase transformation (Figure 1a-f). This can be explained by finite element calculations (Figure 1g-h), which show that indentation into samples with the geometry used during in-situ nanoindentation has a different stress condition, resulting in favorable conditions for surface nucleation. ${ }^{7}$

Similarly, dislocation plasticity in silicon results when diamond-like carbon films are used to protect the silicon. We have looked at the effect of both "amorphous diamond" and ultrananocrystalline diamond films on silicon. ${ }^{9}$ In each case, the films do not show significant permanent deformation, while the underlying silicon deforms plastically through dislocation nucleation and glide.

References:

[1] M. Wall and U. Dahmen, Microscopy and Microanalysis, 3 (1997) pp. 593-4.

[2] E.A. Stach, et al., Micr. and Microanal. 7, (2001) pp. 507-17.

[3] A.M. Minor, J.W. Morris, Jr., E.A. Stach, Appl. Phys. Let. 79, (2001) pp. 1625-7.

[4] A.M. Minor, E.T. Lilleodden, E.A. Stach and J.W. Morris, Jr, J. Elect. Mat., in press, 2003.

[5] E.T. Lilleodden, Ph.D. dissertation, Stanford University, 2002.

[6] See e.g. V. Domnich and Y. Gogotsi, Rev. Adv. Mat. Sci., 3 (2002) pp.1-36.

[7] A.M. Minor ${ }^{2}$, E.T. Lilleodden ${ }^{2}$, M. Jin ${ }^{2,3}$, E.A. Stach ${ }^{1}$, D. Chrzan ${ }^{2,3}$, J.W. Morris, Jr., submitted, 2003.

[8] TA Friedmann, et al. Appl. Phys. Lett. 68 (1996) pp.1643-5.

[9] D. Zhou, et al. J. Appl. Phys. 82 (1997) pp.4546-4550.

[10] This work was supported by the U.S. Department of Energy under Contract No. DE-AC03-76SF00098. 


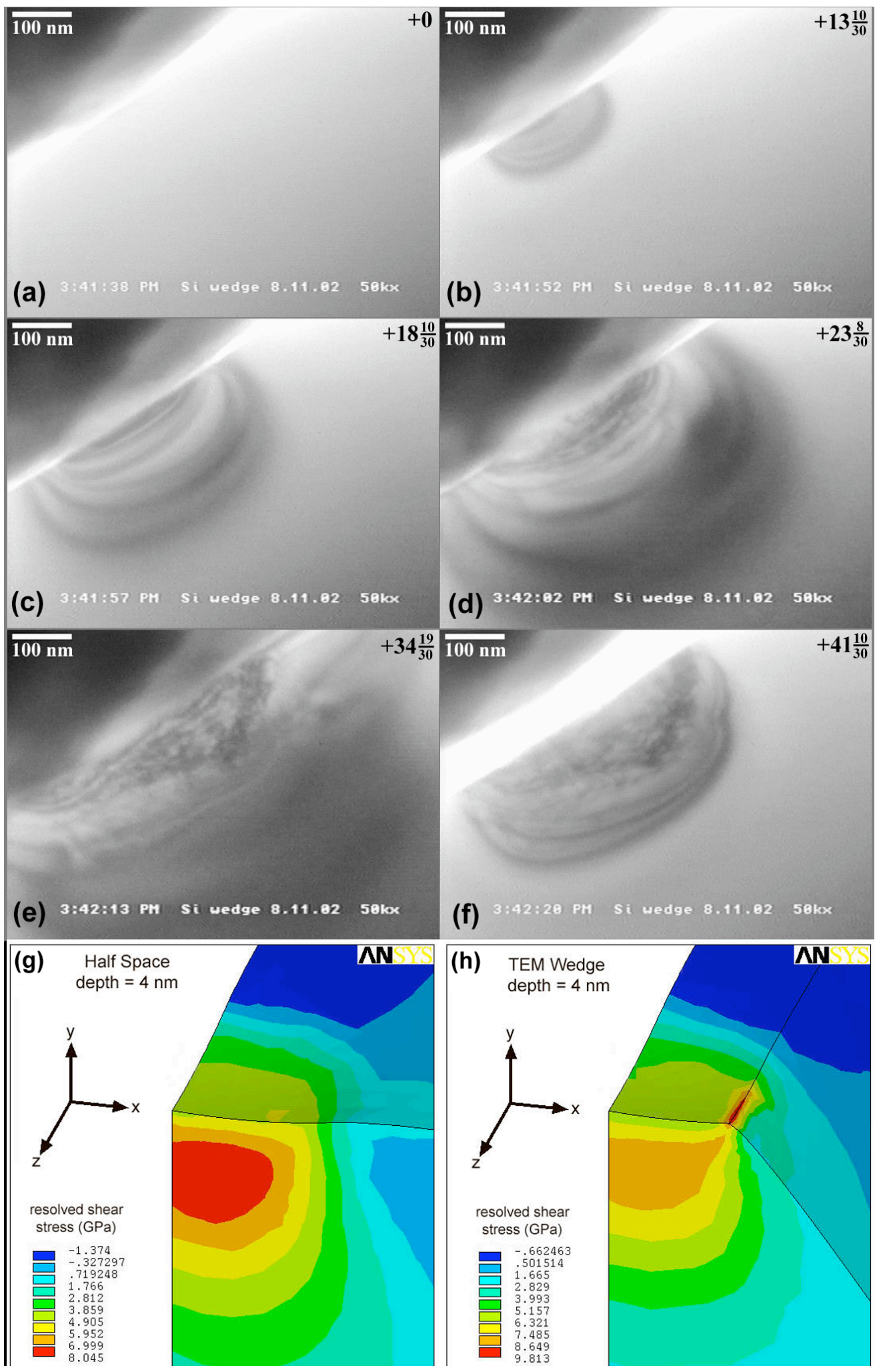

Figure 1 - Video frames from the in-situ nanoindentation of single crystal silicon showing the evolution of elastic (a-c) and plastic (d-f) deformation. Finite element models (g\&h) show are used to show how the sample geometry results in a change of stress in the sample, making surface nucleation easier. 